\title{
Inhibition of Cytochrome P450 by Propolis in Human Liver Microsomes
}

\author{
Chang Seon Ryu ${ }^{1,+}$, Soo Jin $\mathrm{Oh}^{2,+}$, Jung Min Oh', Ji-Yoon Lee', Sang Yoon Lee', \\ Jung-woo Chae ${ }^{1}$, Kwang-il Kwon ${ }^{1}$ and Sang Kyum Kim ${ }^{1}$ \\ ${ }^{1}$ College of Pharmacy, Chungnam National University, Daejeon, Korea \\ ${ }^{2}$ Bio-Evaluation Center, Korea Research Institute of Bioscience and Biotechnology, Ochang, Korea
}

(Received April 15, 2016; Revised May 20, 2016; Accepted May 23, 2016)

\begin{abstract}
Although propolis is one of the most popular functional foods for human health, there have been no comprehensive studies of herb-drug interactions through cytochrome P450 (CYP) inhibition. The purpose of this study was to determine the inhibitory effects of propolis on the activities of CYP1A2, 2A6, 2B6, 2C9, 2C19, 2D6, 2E1 and 3A4 using pooled human liver microsomes (HLMs). Propolis inhibited CYP1A2, CYP2E1 and CYP2C19 with an $\mathrm{IC}_{50}$ value of $6.9,16.8$, and $43.1 \mu \mathrm{g} / \mathrm{mL}$, respectively, whereas CYP2A6, 2B6, 2C9, 2D6, and 3A4 were unaffected. Based on half-maximal inhibitory concentration shifts between microsomes incubated with and without nicotinamide adenine dinucleotide phosphate, propolis-induced CYP1A2, CYP2C19, and CYP2E1 inhibition was metabolism-independent. To evaluate the interaction potential between propolis and therapeutic drugs, the effects of propolis on metabolism of duloxetine, a serotonin-norepinephrine reuptake inhibitor, were determined in HLMs. CYP1A2 and CYP2D6 are involved in hydroxylation of duloxetine to 4-hydroxy duloxetine, the major metabolite, which was decreased following propolis addition in HLMs. These results raise the possibility of interactions between propolis and therapeutic drugs metabolized by CYP1A2.
\end{abstract}

Key words: Cytochrome P450, Propolis, Drug-drug interaction, Duloxetine

\section{INTRODUCTION}

As the elderly population increases, the demand for, and consumption of, both functional foods and medical drugs also increases. Thus, functional foods and medical drugs may be simultaneously administered in the elderly population suffering from chronic disease. Because some components of functional foods are eliminated from the body by the same mechanisms that remove drugs, the potential exists for interaction between functional food components and drugs (1). In fact, various herbal products inhibit or induce activity of hepatic cytochrome P450 (CYP) isoforms, which play a critical role in elimination of xenobiot-

Correspondence to: Sang Kyum Kim, College of Pharmacy, Chungnam National University, 220 Gung-dong, Yuseong-gu, Daejeon 34134, Korea

E-mail: sangkim@cnu.ac.kr

${ }^{\dagger}$ These authors contributed equally to this work.

This is an Open-Access article distributed under the terms of the Creative Commons Attribution Non-Commercial License (http:// creativecommons.org/licenses/by-nc/3.0) which permits unrestricted non-commercial use, distribution, and reproduction in any medium, provided the original work is properly cited. ics via metabolism (2).

CYP comprises a superfamily of enzymes involved in the oxidation of a great number of exogenous and endogenous compounds. Inhibition of human CYPs is one of the most common mechanisms leading to drug-drug interactions. Among the numerous human hepatic CYP enzymes identified to date, eight CYPs (CYP1A2, 2A6, 2B6, 2C9, 2C19, 2D6, 2E1 and 3A4) play dominant roles in the metabolism of drugs and other xenobiotics (3). Our previous studies showed that Ssang-hwa-tang and Galgeun-tang inhibited CYP1A2 in human liver microsomes (HLMs), and induced CYP1B1, CYP2E1 and CYP4A1 in rats $(4,5)$.

Propolis is one of the most popular functional foods used to improve human health globally. Propolis is a multifunctional material used by honeybees in beehive construction and maintenance. Propolis is a naturopathic formulation collected by honeybees from buds and exudates of conifer trees and plants. Biological activities of propolis depend mainly upon the chemical composition of the constituents, including volatile organic compounds, flavonoid aglycones, phenolic acids and their esters, phenolic aldehydes, alcohols and ketones, sesquiterpenes, quinones, coumarins, steroids and amino acids (6). Phenolic acids and their esters isolated from propolis exert antioxidant activity. Caffeic 
acid phenethyl ester (CAPE), one of the major biologically active components of propolis, elicited protective effects against erythrocyte membrane lipid peroxidation, cellular strand breakage, and protein fragmentation (7). In addition, propolis exerted a hepatic protective effect in mice treated with acetaminophen through inhibition of CYP activities and induction of phenolsulphotransferase activity (8).

Recently, effects of an ethanol extract of Brazilian green propolis and its major ingredients on CYP1A2, 2C9, 2C19, 2D6 and 3A4 were determined using recombinant human CYP (9). Activities of CYP1A2, 2C9, 2C19, 2D6 and 3A4 were attenuated by the ethanol extract of Brazilian green propolis in a concentration-dependent manner. However, inhibitory effects on other CYP isoforms of propolis were not determined and the inhibitory mechanism remains to be determined. Thus, the purpose of this study was to measure the inhibitory effects of propolis on CYP1A2, 2A6, 2B6, 2C9, 2C19, 2D6, 2E1 and 3A4 activities using pooled HLMs and determine whether the inhibition of CYP enzymes by propolis is metabolism-dependent. Moreover, to evaluate the potential for interactions between propolis and drugs used to treat chronic disease, the effects of propolis on the metabolism of duloxetine, a serotonin-norepinephrine reuptake inhibitor (SNRI), were determined in HLMs.

\section{MATERIALS AND METHODS}

Chemicals and reagents. Duloxetine hydrochloride, apigenin, caffeic acid, chrysin, galangn, kaempferide, kaempferol, $p$-coumaric acid, duloxetine hydrochloride, 4-methylumbelliferone (4MU) and the reduced form of $\beta$-nicotinamide adenine dinucleotide phosphate (NADPH) were purchased from Sigma-Aldrich (St. Louis, MO, USA). 4Hydroxy duloxetine was purchased from Santa Cruz Biotechnology (Santa Cruz, CA, USA). Artepillin C purchased from Wako Pure Chemicals (Osaka, Japan). Pooled human liver microsomes (BD UltraPool ${ }^{\mathrm{TM}}$ HLM 150, lot 38289) were purchased from BD Gentest Co. (Woburn, MA, USA). The manufacturer supplied information regarding the protein concentration, CYP content, and enzyme activity. Propolis capsules were purchased from Natural Immix Health Ltd. (Port Coquitlam, BC, Canada). The ratio of the starting crude material to the powdered extract was 5:1. Propolis capsules contained only propolis extract from bee hive of the honey bee without additives. All other reagents and chemicals were of analytical or HPLC grade.

Quantitative determination of major ingredients in propolis extract. To quantify major ingredients, propolis extract was dissolved to $200 \mu \mathrm{g} / \mathrm{mL}$ in $0.1 \mathrm{M}$ phosphate buffer ( $\mathrm{pH}$ 7.4) containing $0.5 \%$ dimethyl sulfoxide (DMSO). Liquid chromatography electrospray ionization tandem mass spectrometry (LC-MS/MS) was used to develop a method to determine the amount of major ingredients present in
Table 1. Major ingredients and their respective contents in propolis extract

\begin{tabular}{lr}
\hline \hline Ingredients & $(\mu \mathrm{g} / \mathrm{mg})$ \\
\hline Chrysin & $23.57 \pm 3.02$ \\
Galangin & $7.45 \pm 0.51$ \\
Kaempferide & $4.30 \pm 0.57$ \\
Kaempferol & $4.19 \pm 0.27$ \\
Caffeic acid phenethyl ester & $0.35 \pm 0.01$ \\
Apigenin & $0.19 \pm 0.10$ \\
Artepillin C & $0.05 \pm 0.04$ \\
$p$-Coumaric acid & $0.03 \pm 0.01$ \\
Caffeic acid & $0.01 \pm 0.00$ \\
\hline
\end{tabular}

Each value represents the mean \pm SD for three samples.

propolis extract. To separate major ingredients, an $\mathrm{Xterra}^{\mathrm{TM}}$ C18 column $(50 \times 2.1 \mathrm{~mm}$ i.d., $3 \mu \mathrm{m}$; Waters, Milford, MA, USA) with a SecurityGuard ${ }^{\mathrm{TM}}$ C18 guard column $(2.0 \times 4.0$ $\mathrm{mm}$ i.d.; Phenomenex, Torrance, CA, USA) was used with a gradient elution system consisting of deionized water containing $0.1 \% \mathrm{v} / \mathrm{v}$ formic acid and acetonitrile containing $0.1 \%(\mathrm{v} / \mathrm{v})$ formic acid. The major ingredients calibration standard $(0.278-71 \mu \mathrm{g} / \mathrm{mL})$ was prepared under the same conditions as the propolis extract. 4MU was used as an internal standard (IS). Standard component calibration curves fit the linear regression well $\left(\mathrm{R}^{2}>0.9999\right)$. The LC-ESI/MS/ MS system consisted of a Shimadzu 20AD-XR high performance liquid chromatography (HPLC) system (Shimadzu, Kyoto, Japan) and an API 3200Q-TRAP LC-MS/MS system equipped with a Turbo V Ion Spray ${ }^{\mathrm{TM}}$ source (Applied Biosystems, Foster City, CA, USA) operated in the negative ion mode. Selective reaction monitoring of the deprotonated precursor ion and the related product ion was used to quantify major ingredients and 4MU using the IS method with peak area ratios. The quantities of major ingredients are shown in Table 1.

In vitro direct CYP inhibition assay. Metabolites produced by CYP isoforms were determined using mass spectrometry based on previously established $(3,10)$.

In vitro metabolism-dependent CYP inhibition assay. Metabolism- or time-dependent CYP inhibition is mainly caused by metabolite(s) rather than parent drug itself, because CYP-mediated metabolite exhibits more potent inhibitory activity against CYP than the parent compound. Metabolism-dependent inhibition was identified based on previously described method of $\mathrm{IC}_{50}$ shifts between microsomes incubated with and without NADPH (11).

Inhibition of duloxetine metabolism by propolis. The incubation mixtures (final volume, $200 \mu \mathrm{L}$ ) contained 0.5 $\mathrm{mg} / \mathrm{mL}$ microsomal protein, $0.1 \mathrm{M}$ potassium phosphate buffer ( $\mathrm{pH}$ 7.4), an NADPH-generating system mixture 
(final $1 \mathrm{mM}$ NADP, $5 \mathrm{mM}$ glucose-6-phosphate, and 10 unit/mL glucose-6-phosphate dehydrogenase), and 1 or $20 \mu \mathrm{M}$ duloxetine as a substrate. Duloxetine was used at concentrations approximately equal to the $\mathrm{K}_{\mathrm{m}}$ values of 4hydroxy duloxetine, the major metabolite of duloxetine (12). The low or high affinity $\mathrm{K}_{\mathrm{m}}$ values were 1 or $20 \mu \mathrm{M}$, respectively (13). After a $5 \mathrm{~min}$ preincubation at $37^{\circ} \mathrm{C}$ with $30 \mu \mathrm{g} / \mathrm{mL}$ propolis, the reactions were initiated by the addition of the NADPH-generating system mixture and duloxetine. The incubations were performed at $37^{\circ} \mathrm{C}$ for $60 \mathrm{~min}$ in a shaking water bath and terminated by adding $200 \mu \mathrm{L}$ of cold acetonitrile containing $50 \mathrm{nM}$ cabamazepine as an internal standard after a 60 min incubation period, and agitating with a vortex mixer before centrifugation. The samples for each enzyme assay were centrifuged at 2,500 $\times \mathrm{g}$ for $20 \mathrm{~min}$ at $4^{\circ} \mathrm{C}$, and the supernatants of the individual reaction samples were analyzed by LC-MS/MS (14). Compounds were separated on an Atlantis C18 column $(50 \times 2.1 \mathrm{~mm}$ i.d., $3 \mu \mathrm{m}$; Waters) with an isocratic mobile phase consisting of methanol and $5 \mathrm{mM}$ ammonium acetate $(6: 4, \mathrm{v} / \mathrm{v})$. The column was heated to $40^{\circ} \mathrm{C}$, and the mobile phase was eluted at $0.4 \mathrm{~mL} / \mathrm{min}$. Selective reaction monitoring of the protonated precursor ion and the related product ion was used. The mass transitions used for 4-hydroxy duloxetine and carbamazepine were $\mathrm{m} / \mathrm{z} 314>154$, and $237>194$, respectively.

Data analysis. The $\mathrm{IC}_{50}$ was calculated using nonlinear regression of the plot of percent control activity versus test inhibitor concentration. All results expressed as means $\pm \mathrm{SD}$ were analyzed using Student's $t$-tests (Graph Pad Prism 5.0, San Diego, CA, USA). The acceptable level of significance was established at $P<0.05$, except where otherwise indicated.
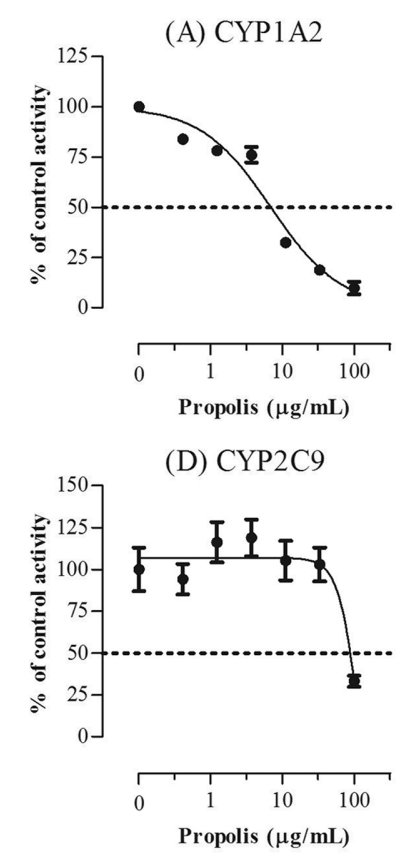

(G) CYP2E1

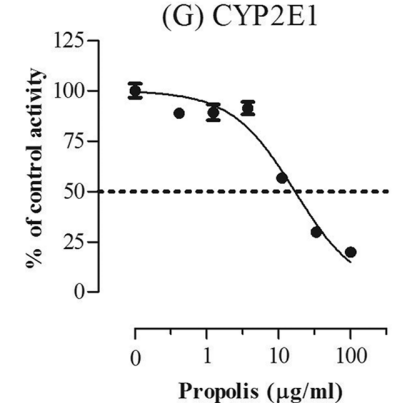

(B) CYP2A6

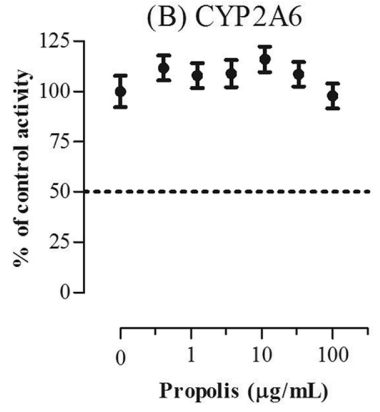

(E) $\mathrm{CYP} 2 \mathrm{C} 19$

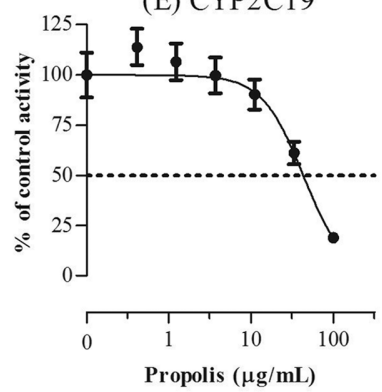

(H) CYP3A4(M)

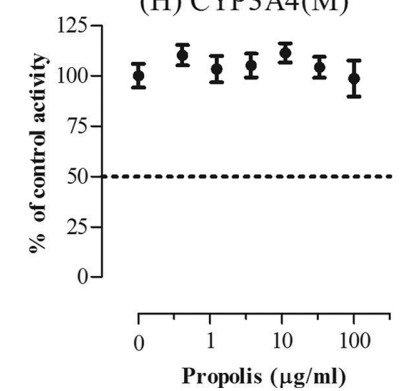

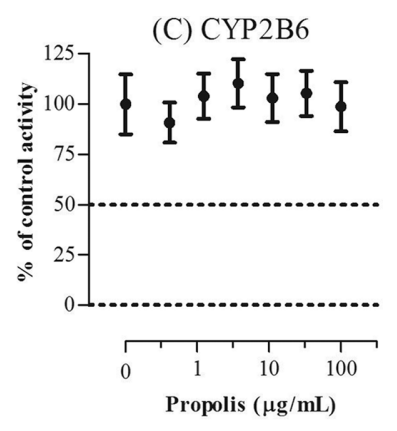

(F) CYP2D6

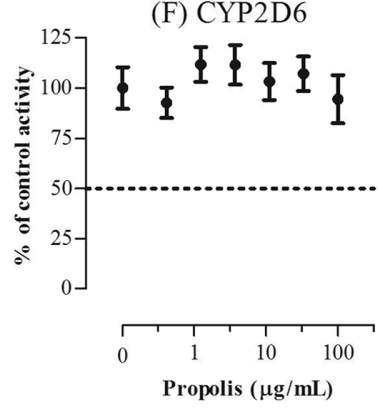

(I) CYP3A4(T)

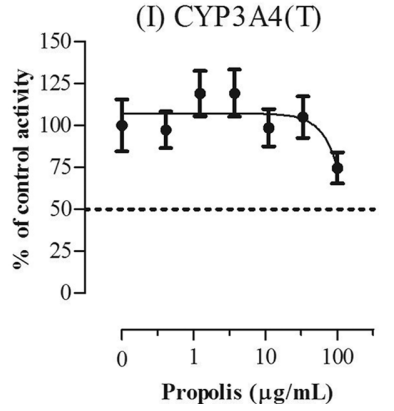

Fig. 1. Inhibitory effects of propolis on cytochrome P450 (CYP)1A2 (A), CYP2A6 (B), CYP2B6 (C), CYP2C9 (D), CYP2C19 (E), CYP2D6 (F), CYP2E1 (G), CYP3A4 (midazolam as a substrate) (H), and CYP3A4 (testosterone as a substrate) (I) in pooled human liver microsomes (HLMs). Activity is expressed as the percentage of activity remaining as compared with a control sample containing no propolis $(100 \%)$. Each data point represents the mean \pm SD of three separate samples. 


\section{RESULTS}

Direct CYP inhibition by propolis. The inhibitory potential of propolis on the formation of metabolites by eight CYP isoforms was evaluated using pooled HLMs and substrate cocktails. Experimental $\mathrm{IC}_{50}$ values of propolis were determined from seven-point concentration-response curves (Fig. 1). Ketoconazole was used as a positive control for CYP3A4 inhibition and showed strong activity with an $\mathrm{IC}_{50}$ value within the reference range $(0.21 \mu \mathrm{M}$ using
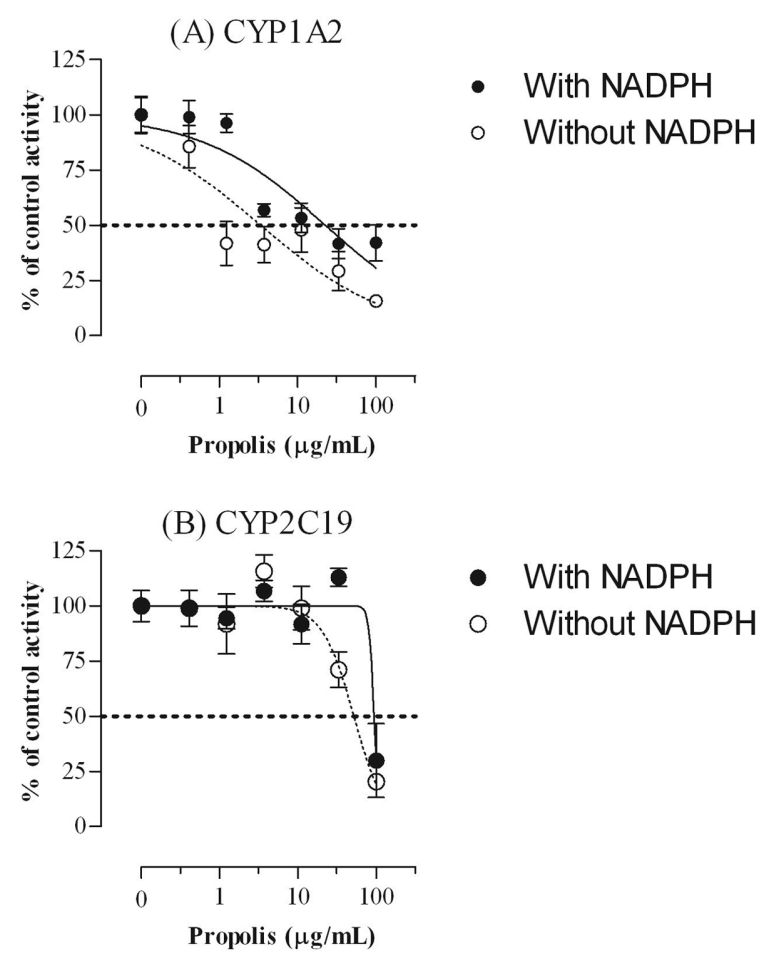

- With NADPH

- Without NADPH

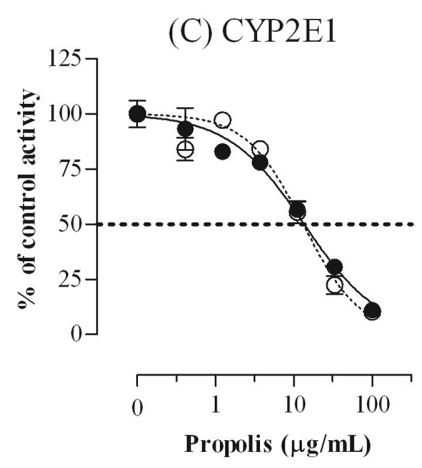

- With NADPH

- Without NADPH

Fig. 2. Changes in the inhibition curves of CYP1A2 (A), CYP2C19 (B) and CYP2E1 by propolis after HLM preincubation with nicotinamide adenine dinucleotide phosphate (NADPH) for $30 \mathrm{~min}$. HLMs were incubated with propolis for $30 \mathrm{~min}$ in the presence or absence of NADPH. Activity is expressed as the percentage of activity remaining as compared with a control sample containing no propolis (100\%). Data show the mean $\pm \%$ SD of three separate samples. midazolam as a substrate and $0.10 \mu \mathrm{M}$ using testosterone as a substrate). $\mathrm{IC}_{50}$ values ( $95 \%$ confidence intervals, $\mathrm{CI}$ ) of propolis against CYP1A2, CYP2C19, and CYP2E1 were $6.88 \mu \mathrm{g} / \mathrm{mL}$ (3.54 to $13.36 \mu \mathrm{g} / \mathrm{mL}$ ), $43.14 \mu \mathrm{g} / \mathrm{mL}$ (28.18 to $66.06 \mu \mathrm{g} / \mathrm{mL}$ ), and $16.77 \mu \mathrm{g} / \mathrm{mL}$ (9.56 to $29.42 \mu \mathrm{g} / \mathrm{mL}$ ), respectively. CYP2C9 activity was decreased to $33 \%$ of the control level by $100 \mu \mathrm{g} / \mathrm{mL}$ propolis (Fig. 1D). CYP2A6, 2B6, 2D6 and 3A4 were not markedly inhibited by propolis at concentrations up to $100 \mu \mathrm{g} / \mathrm{mL}$ (Fig. 1).

Mechanism of propolis-induced CYP1A2, 2C19 and 2E1 inhibition. To determine the mechanism underlying the inhibitory activity of propolis on CYP1A2, CYP2C19 and CYP2E1, the metabolism-dependent inhibitory potential of propolis was evaluated. Furafylline, which was used as a positive control for the metabolism-dependent inhibition of CYP1A2, showed strong activity with both the concentration of inhibitor which gives half the maximal rate of inactivation $\left(\mathrm{K}_{\mathrm{I}}\right)$ and maximal rate of enzyme inactivation at a saturating concentration of inhibitor $\left(\mathrm{k}_{\text {inact }}\right)$ values within the reference range (data not shown) (4). The propolis $\mathrm{IC}_{50}$ shifts on CYP1A2 and 2E1 were 0.11 and 0.98 , respectively, showing that the inhibitory effects of propolis on CYP1A2 were markedly attenuated by preincubation with NADPH (Fig. 2). The propolis $\mathrm{IC}_{50}$ values against CYP2C19 in HLMs preincubated with NADPH and propolis were not calculable, because the CYP2C19 inhibition by propolis was observed only at the maximal concentration of propolis $(100 \mu \mathrm{g} / \mathrm{mL})$ preincubated with HLM in the presence of NADPH (Fig. 2B). Thus, the $\mathrm{IC}_{50}$ fold-shifts of

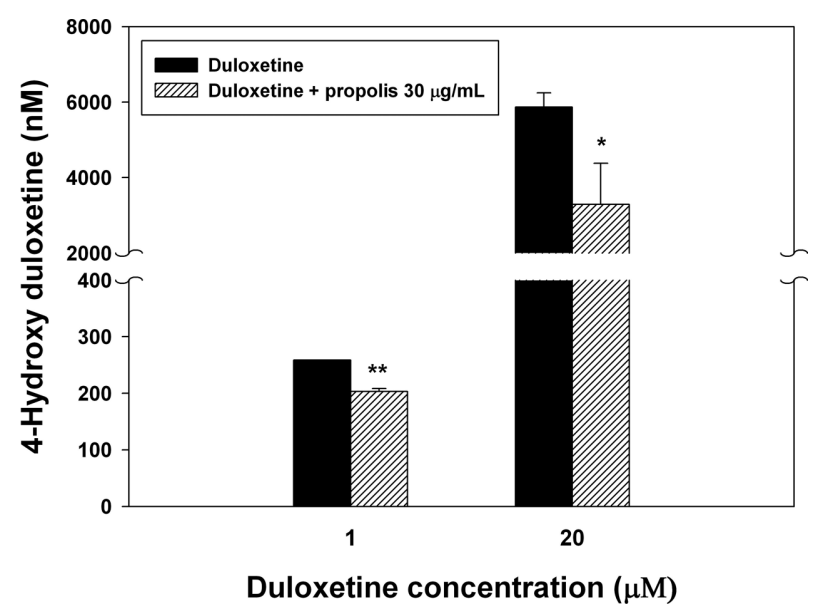

Fig. 3. Inhibitory effects of propolis on duloxetine metabolism in pooled HLMs. 4-Hydroxy duloxetine, the major metabolite of duloxetine, was determined using LC-MS/MS. Duloxetine (1 or $20 \mu \mathrm{M})$ was incubated with pooled HLMs in the presence or absence of propolis $(30 \mu \mathrm{g} / \mathrm{mL})$. Each data point represents the mean \pm SD of three separate samples. ${ }^{* * *}$ Significantly different from the control sample incubated with duloxetine only, $p<$ 0.05 or $p<0.01$, respectively (Student's $t$-test). 
propolis calculated by a without NADPH/with NADPH ratio did not exceed 1.5-fold, suggesting that the propolis-mediated CYP1A2, CYP2C9 and CYP2E1 inhibition was metabolism-independent.

Inhibition of duloxetine metabolism by propolis. To evaluate the potential for interactions between propolis and drugs used to treat chronic disease, the effects of propolis on metabolism of duloxetine, a SNRI, were determined in HLMs. 4-Hydroxy duloxetine, the major metabolite of duloxetine produced by CYP1A2 and CYP2D6, was determined in HLMs in the presence of $30 \mu \mathrm{g} / \mathrm{mL}$ propolis (Fig. $3)$. The formation of 4-hydroxy duloxetine from duloxetine (1 or $20 \mu \mathrm{M}$ ) was decreased to 78 or $56 \%$ of control level, respectively, by $30 \mu \mathrm{g} / \mathrm{mL}$ propolis.

\section{DISCUSSION}

Effects of propolis extract on CYP-mediated metabolic activities were determined using HLMs. The results showed that propolis had potent CYP1A2, CYP2C19 and CYP2E1 inhibitory activity and weak CYP2C9 inhibitory activity, based on the $\mathrm{IC}_{50}$ values. These results showing strong inhibitory activity of propolis extract against CYP1A2 and CYP2C19 were consistent with previous results reported by Naramoto (9). In the present study, CYP2C9, 2D6 and CYP3A4 were not markedly inhibited by propolis, which is different from the previous study (9). The difference in inhibitory effects against CYP enzymes between propolis extracts may reflect differences in ingredients of each extract. Chemical composition of propolis is dependent on the origin of the plants from which the resin is collected and the species of bees $(15,16)$.

Chrysin, one of major flavone components of propolis, showed inhibitory effects on 2,3,7,8-tetrachlorodibenzodioxin-induced CYP1A1 activity in Caco-2 cells (17). Moreover, chrysin, is an effective inhibitor of midazolam 4hydroxylase in HLMs (18). Also CAPE, one of the major biologically active components of propolis, had CYP1A2 inhibitory activity in HLM (19) and diminished hepatic protein levels of CYP1A2 and CYP2B1/2 in rats (20). Also, CAPE inhibits 3-methylcholanthrene-induced CYP1A1 expression through induction of hypoxia-inducible factor-1 (21). These results suggest the role of chrysin and CAPE in propolis-mediated CYP inhibition. However, these results do not rule out the possibility that other propolis components inhibit CYP activities in HLMs. For example, artepillin $\mathrm{C}$, galangin, apigenin, kaempferol, isosakuranetin and kaempferide observed in propolis exhibits showed inhibitory activities against some CYP isoforms including CYP1A2, CYP2C9 and CYP3A4 $(9,22,23)$.

The metabolism-dependent inhibition of CYP isoforms can be identified based on a shift in the $\mathrm{IC}_{50}$ value after a 30 min preincubation of the tested compounds with NADPH.
In the present study, propolis preincubation in HLMs with NADPH markedly attenuated the inhibitory effects of propolis on CYP1A2 and CYP2C19. In contrast, CYP2E1 inhibition by propolis was unaffected by preincubation with $\mathrm{NADPH}$, based on $\mathrm{IC}_{50}$ shifts. The mechanism underlying the attenuation by preincubation with NADPH remains to be determined, but it may be due to the change in propolis chemical composition during the propolis extract incubation in HLMs with NADPH. An analysis of the quantitative structure-activity relationship using inhibition data of 16 naturally occurring flavonoids postulated that the CYP1A2 inhibitory effect was governed by factors such as the number of hydroxyl groups (24). These authors suggested that an increase in the number of free hydroxyl groups decreased the inhibitory effect on CYP1A2 activity. Mono-oxygenation, the major NADPH-dependent reaction in HLMs, can introduce hydroxyl groups to the A and B ring of flavonoids found in propolis and consequently attenuate the inhibitory effects of flavonoids on CYP1A2.

Various functional foods have the potential to inhibit or induce drug-metabolizing enzymes, particularly CYPs (25). Consequently, drug-drug interactions can be observed in patients using pharmaceutical drugs in combination with functional foods. In the present study, the interaction potential between propolis and duloxetine was determined in HLMs. Duloxetine is a SNRI prescribed for depressive disorders and neuropathic pain. Duloxetine undergoes hepatic metabolism, predominantly through CYP2D6 and CYP1A2, and 4-hydroxy duloxetine is the major metabolite. In the present study the formation of 4-hydroxy duloxetine was significantly decreased by $30 \mu \mathrm{g} / \mathrm{mL}$ propolis. These results raise the possibility that propolis has the drug interaction potential with drugs used to treat chronic diseases. To evaluate the possibility of drug-drug interactions, the applied dose and the pharmacokinetic properties of the components with inhibitory activity must be considered in addition to the in vitro inhibitory potency of functional foods.

CYP1A2 is important in the metabolism of frequently used drugs, including theophylline, acetaminophen, propranolol and a number of antidepressants (26). Fluvoxamine, furafylline, quinolone antibiotics and oral contraceptives are CYP1A2 inhibitors $(27,28)$. CYP1A2 inhibition is particularly important if a drug has a narrow therapeutic index prior to the appearance of toxicity and adverse effects. Duloxetine is a sensitive in vivo substrate of CYP1A2 that represents drugs whose plasma area under the curve (AUC) values increase 5 -fold or higher when they are co-administered with a known CYP inhibitor (29). Thus, decreased duloxetine metabolism by propolis can be attributed to CYP1A2 inhibition.

CYP2C19 is one of the major CYP isoforms involved in the metabolism of anticonvulsants, antiplatelet drugs, proton pump inhibitors and tricyclic antidepressants (30). Thus, evaluation of potential CYP2C19 inhibition is essential in 
the drug development and discovery process. In the present study, the propolis $\mathrm{IC}_{50}$ value against CYP2C19 was 43.14 $\mu \mathrm{g} / \mathrm{mL}$, and HLM preincubation markedly attenuated the CYP2C19 inhibition by propolis. Thus, propolis-induced CYP1A2 inhibition may be more potent than its CYP2C19 inhibition.

Although CYP2E1 is considered to be a minor CYP isoform involved in pharmaceutical drug metabolism, this enzyme plays a major role in environmental pollutant metabolism. CYP2E1 can also metabolize endogenous compounds such as hydroxyeicosatetraenoic acids, and thus, CYP2E1 is involved in metabolism of arachidonic acid (31). The inhibitory effects of propolis on CYP2E1 can change the metabolism of endogenous compounds as well as xenobiotics. These results raise the possibility that CYP2E1 inhibition may be responsible for the hepatoprotective effects of propolis in mice treated with acetaminophen (8) and in rat treated with carbon tetrachloride (32).

In conclusion, propolis was found to exert potent CYP1A2 and CYP2E1 inhibitory activity determined using HLMs in a metabolism-independent manner. Moreover, the metabolism of duloxetine to 4-hydroxy duloxetine in HLMs was decreased following propolis addition, which raises the possibility of interactions between propolis and medicinal drugs metabolized by CYP1A2. Further studies should focus on the identification of CYP regulatory component(s) from propolis and the in vivo evaluation of the metabolic interaction between propolis and duloxetine to confirm the present results.

\section{ACKNOWLEDGMENTS}

This work was supported by research fund of Chungnam National University.

\section{INTEREST OF CONFLICTS}

The authors declare that they have no competing interests.

\section{REFERENCES}

1. Venkataramanan, R., Komoroski, B. and Strom, S. (2006) In vitro and in vivo assessment of herb drug interactions. Life Sci., 78, 2105-2015.

2. Na, D.H., Ji, H.Y., Park, E.J., Kim, M.S., Liu, K.H. and Lee, H.S. (2011) Evaluation of metabolism-mediated herb-drug interactions. Arch. Pharm. Res., 34, 1829-1842.

3. Lee, K.S. and Kim, S.K. (2013) Direct and metabolismdependent cytochrome P450 inhibition assays for evaluating drug-drug interactions. J. Appl. Toxicol., 33, 100-108.

4. Lee, S.Y., Lee, J.Y., Kang, W., Kwon, K.I., Oh, S.J., Ma, J.Y. and Kim, S.K. (2013) In vitro and in vivo assessment of cytochrome P450-mediated herb-drug interaction of Ssang-hwatang. Food Chem., 136, 450-457.

5. Lee, S.Y., Lee, J.Y., Kang, W., Kwon, K.I., Park, S.K., Oh, S.J., Ma, J.Y. and Kim, S.K. (2013) Cytochrome P450-medi- ated herb-drug interaction potential of Galgeun-tang. Food Chem. Toxicol., 51, 343-349.

6. Sobocanec, S., Sverko, V., Balog, T., Sarić, A., Rusak, G., Likić, S., Kusić, B., Katalinić, V., Radić, S. and Marotti, T. (2006) Oxidant/antioxidant properties of Croatian native propolis. J. Agric. Food Chem., 54, 8018-8026.

7. Wang, T., Chen, L., Wu, W., Long, Y. and Wang, R. (2008) Potential cytoprotection: antioxidant defense by caffeic acid phenethyl ester against free radical-induced damage of lipids, DNA, and proteins. Can. J. Physiol. Pharmacol., 86, 279-287.

8. Seo, K.W., Park, M., Song, Y.J., Kim, S.J. and Yoon, K.R. (2003) The protective effects of Propolis on hepatic injury and its mechanism. Phytother. Res., 17, 250-253.

9. Naramoto, K., Kato, M. and Ichihara, K. (2014) Effects of an ethanol extract of Brazilian green propolis on human cytochrome $\mathrm{P} 450$ enzyme activities in vitro. J. Agric. Food Chem., 62, 11296-11302.

10. Lee, S.Y., Jang, H., Lee, J.Y., Kwon, K.I., Oh, S.J. and Kim, S.K. (2014) Inhibition of cytochrome P450 by ethambutol in human liver. Toxicol. Lett., 229, 33-40.

11. Lee, J.Y., Lee, S.Y., Oh, S.J., Lee, K.H., Jung, Y.S. and Kim, S.K. (2012) Assessment of drug-drug interactions caused by metabolism-dependent cytochrome P450 inhibition. Chem. Biol. Interact., 198, 49-56.

12. Lantz, R.J., Gillespie, T.A., Rash, T.J., Kuo, F., Skinner, M., Kuan, H.Y. and Knadler, M.P. (2003) Metabolism, excretion, and pharmacokinetics of duloxetine in healthy human subjects. Drug Metab. Dispos., 31, 1142-1150.

13. Lobo, E.D., Bergstrom, R.F., Reddy, S., Quinlan, T., Chappell, J., Hong, Q., Ring, B. and Knadler, M.P. (2008) In vitro and in vivo evaluations of cytochrome P450 1A2 interactions with duloxetine. Clin. Pharmacokinet., 47, 191-202.

14. Chae, J.W., Baek, H.M., Kim, S.K., Kang, H.I. and Kwon, K.I. (2013) Quantitative determination of duloxetine and its metabolite in rat plasma by HPLC-MS/MS. Biomed. Chromatogr., 27, 953-955.

15. Ahn, M.R., Kumazawa, S., Usui, Y., Nakamura, J., Matsuka, M., Zhu, F. and Nakayama, T. (2007) Antioxidant activity and constituents of propolis collected in various areas of China. Food Chem., 101, 1383-1392.

16. Sibel, S. and Semiramis, K. (2005) Chemical composition and antibacterial activity of propolis collected by three different races of honeybees in the same region. J. Ethnopharmacol., 99, 69-73.

17. Sergent, T., Dupont, I., Van der Heiden, E., Scippo, M.L., Pussemier, L., Larondelle, Y. and Schneider, Y.J. (2009) CYP1A1 and CYP3A4 modulation by dietary flavonoids in human intestinal Caco-2 cells. Toxicol. Lett., 191, 216-222.

18. Quintieri, L., Palatini, P., Nassi, A., Ruzza, P. and Floreani, M. (2008) Flavonoids diosmetin and luteolin inhibit midazolam metabolism by human liver microsomes and recombinant CYP 3A4 and CYP3A5 enzymes. Biochem. Pharmacol., 75, 1426-1437.

19. Jaikang, C., Chaiyasut, C., Narongchai, P., Niwatananun, K., Narongchai, S. and Kusirisin, W. (2011) Inhibitory effects of caffeic acid ester analogues on free radicals and human liver microsome CYP1A2 activities. Med. Chem., 7, 99-105.

20. Beltrán-Ramírez, O., Pérez, R.M., Sierra-Santoyo, A. and Villa-Treviño, S. (2012) Cancer prevention mediated by caf- 
feic acid phenethyl ester involves cyp2b1/2 modulation in hepatocarcinogenesis. Toxicol. Pathol., 40, 466-472.

21. Kim, H.G., Han, E.H., Im, J.H., Lee, E.J., Jin, S.W. and Jeong, H.G. (2015) Caffeic acid phenethyl ester inhibits 3-MCinduced CYP1A1 expression through induction of hypoxiainducible factor-1 $\alpha$. Biochem. Biophys. Res. Commun., 465, 562-568.

22. Kimura, Y., Ito, H., Ohnishi, R. and Hatano, T. (2010) Inhibitory effects of polyphenols on human cytochrome P450 3A4 and 2C9 activity. Food Chem. Toxicol., 48, 429-435.

23. Shimada, H., Eto, M., Ohtaguro, M., Ohtsubo, M., Mizukami, Y., Ide, T. and Imamura, Y. (2010) Differential mechanisms for the inhibition of human cytochrome P450 1A2 by apigenin and genistein. J. Biochem. Mol. Toxicol., 24, 230-234.

24. Lee, H., Yeom, H., Kim, Y.G., Yoon, C.N., Jin, C., Choi, J.S., Kim, B.R. and Kim, D.H. (1998) Structure-related inhibition of human hepatic caffeine N3-demethylation by naturally occurring flavonoids. Biochem. Pharmacol., 55, 1369-1375.

25. Schmidt, L.E. and Dalhoff, K. (2002) Food-drug interactions. Drugs, 62, 1481-1502.

26. Faber, M.S., Jetter, A. and Fuhr, U. (2005) Assessment of CYP1A2 activity in clinical practice: why, how, and when? Basic Clin. Pharmacol. Toxicol., 97, 125-134.

27. Fuhr, U., Anders, E.M., Mahr, G., Sörgel, F. and Staib, A.H. (1992) Inhibitory potency of quinolone antibacterial agents against cytochrome P450IA2 activity in vivo and in vitro. Antimicrob. Agents Chemother, 36, 942-948.

28. Christensen, M., Tybring, G., Mihara, K., Yasui-Furokori, N., Carrillo, J.A., Ramos, S.I., Andersson, K., Dah, M.L. and Bertilsson, L. (2002) Low daily 10-mg and 20-mg doses of fluvoxamine inhibit the metabolism of both caffeine (cytochrome P4501A2) and omeprazole (cytochrome P4502C19). Clin. Pharmacol. Ther., 71, 141-152.

29. U.S. Department of Health and Human Services, Food and Drug Administration, Center for Drug Evaluation and Research (CDER) (2012) FDA guidacnce for industry. Drug Interaction Studies - Study Design, Data Analysis, Implications for Dosing, and Labeling Recommendations.

30. Foti, R.S. and Wahlstrom, J.L. (2008) CYP2C19 inhibition: the impact of substrate probe selection on in vitro inhibition profiles. Drug Metab. Dispos., 36, 523-528.

31. Laethem, R.M., Balazy, M., Falck, J.R., Laethem, C.L. and Koop, D.R. (1993) Formation of 19(S)-, 19(R)-, and 18(R)hydroxyeicosatetraenoic acids by alcohol-inducible cytochrome P450 2E1. J. Biol. Chem., 268, 12912-12918.

32. Bhadauria, M., Nirala, S.K. and Shukla, S. (2007) Propolis protects CYP 2E1 enzymatic activity and oxidative stress induced by carbon tetrachloride. Mol. Cell. Biochem., 302, 215-224. 